\title{
The Leray-Schauder Degree as Topological Method Solution of Nonlinear Elliptic Equations
}

\author{
Nedal Hassan Elbadowi Eljaneid \\ Department of Mathematics, Faculty of Sciences, University of Tabuk, Tabuk, Kingdom of Saudi Arabia
}

\section{Email address:}

needhal@hotmail.com

\section{To cite this article:}

Nedal Hassan Elbadowi Eljaneid. The Leray-Schauder Degree as Topological Method Solution of Nonlinear Elliptic Equations. Pure and Applied Mathematics Journal. Vol. 6, No. 5, 2017, pp. 148-153. doi: 10.11648/j.pamj.20170605.13

Received: August 11, 2017; Accepted: September 9, 2017; Published: October 29, 2017

\begin{abstract}
In the present paper using precise results on the solutions of linear elliptic differential operators with Holder continuous coefficient as well as a variant of the Lery - Schauder method and the gal of this paper to find an adequate degree theory for the infinite dimensional setting and to extend the theory of homotopy classes of maps form $\mathbb{R}^{n}$ to $\mathbb{R}^{n}$ to homotopy classes of maps on infinite dimensional spaces.
\end{abstract}

Keywords: The Leray - Schauder Degree, Elliptic Boundary Value Problem

\section{Introduction}

The infinite dimensional space under consideration in this section are normed linear vector spaces are their subsets. Extensions will be discussed in the next section. Let $X$ be (a real) linear vector space. On $X$ we define a norm $\|.\|_{X}$ or $\|$. $\|$ for short which satisfies the following hypotheses:

$$
\begin{gathered}
\|x+y\| \leq\|x\|+\|y\| \text {, for all } x, y \in X \\
\|\lambda x\| \leq|\lambda|\|x\| \text {, for all } x \in X \text {, and for all } \lambda \in \mathbb{R} \\
\|x\|=0 \text { if and only if } x=0
\end{gathered}
$$

The combination is called a normed linear vector space. If an addition is $X$ complete it is called is a Banach space. A normed linear space is complete if every Cauchy sequence has a limit in $X ;\left\{x^{n}\right\} \subset X$ with $\left\|x^{n}-x^{m}\right\| \rightarrow 0$ as $n, m \rightarrow$ $\infty$, implies that there exists $a x \in X$ such that $\left\|x^{n}-x\right\| \rightarrow$ 0 as $n \rightarrow \infty$. Normed vector space and Banach space are examples of metric and complete metric spaces respectively, where the metric is given by: $d(x, y)=|x-y|$.

Continuity: Definition (1-1): A mapping $f: X \rightarrow Y$ is continuous if $x^{n} \rightarrow x$ (in $X$ ) implies that $f\left(x^{n}\right) \rightarrow$ $f(x)($ in $Y)$. A map is uniformly continuous on $X$, if for any $\epsilon>0$ there exists $a \delta_{\epsilon}>0$ such that $\|x-y\|<\delta$ implies that $\|f(x)-f(y)\|<\epsilon$. The latter can also be defined with respect to a closed subset $A \subset X$.

A continuous function $f: X \rightarrow Y$ is bounded if $f(\grave{\Omega}) \subset X$


mapping on $\mathbb{R}^{n}$ are necessarily bounded, i.e. bounded sets in $\mathbb{R}^{n}$ are mapped to bounded set under $f$. This is however not the case in general Banach spaces.

Lemma (1-2): A uniformly continuous map is bounded.

Proof: We need to show that for any bounded set the image $A \subset X$ the image $f(A) \subset Y$ is also bounded. Choose $R>0$ such that $A \subset B_{R}(0)$, and let $n>\frac{2 R}{\delta}$. Then for any two points $x, y \in A$ it holds that $\|x-y\| \leq 2 R$, and one can define the line segmen $\mathrm{t}=x+t(y-x), t \in[0,1]$, in $B_{R}(0)$. For $t_{i}=\frac{i}{n}$ we obtain point $x^{t_{i}} \subset B_{R}(0)$, with $\| x^{t_{i}}-$ $x^{t_{i+1}} \|<\delta$, by the choice of $n$. Since $f$ is uniformly continuous it follows that $\left\|f\left(x^{t_{i}}\right)-f\left(x^{t_{i+1}}\right)\right\|<\epsilon$, for all $i$. Form the triangle inequality we then get: $\|f(x)-f(y)\| \leq$ $\sum_{i}\left\|f\left(x^{t_{i}}\right)-f\left(x^{t_{i+1}}\right)\right\|<n \epsilon$.

Which prove the boundedness of $f$ \#

Differentiability: Definition (1-3): A mapping $f C(X, Y)$ is called Gateaux differentiable in the direction $h \in X$, at a point $x_{0}$, if there exists a $y \in Y$ such that $\lim _{t \leftarrow 0}\left\|f\left(x_{0}+t h\right)-f\left(x_{0}\right)-t y\right\|=0$,

With $x_{0}+$ th defined in a neighborhood $N$ of $x_{0}$

Compact and finite rank maps: An important subspace of continuous mappings are the compact mappings from $f: X \rightarrow X$. A mapping $f: X \rightarrow X$ is compact if $\overline{f(\grave{\Omega})}$ is compact for any bounded subset $\grave{\Omega} \subset X$. Compact mapping are bounded since $\overline{f(\grave{\Omega})}$ is bounded for any bounded set $\grave{\Omega} \subset X$. The space of compact mappings on $X$ is denoted by 
$K(X)$.

Definition (1-4): A continuous map K $\Omega \subset X \rightarrow X$ is called compact if $\overline{f(\bar{\Omega})}$ is compact.

Lemma (1-5): Let $\mathrm{k} \in \mathrm{K}(\bar{\Omega})$, then for any, there exists a finite rank map

$\mathrm{k}^{\epsilon} \in \mathrm{F}(\bar{\Omega})$ such that $\left\|\mathrm{k}-\mathrm{k}^{\epsilon}\right\|_{C_{b}^{0}}<\epsilon$ i.e. $\mathrm{k}^{\epsilon} \in \mathrm{F}(\bar{\Omega}) \cap$ $C_{b}^{0}(\bar{\Omega})$

Proof: Let $\overline{k(\bar{\Omega})}$ is compact it can be covered by finitely many balls $B_{\epsilon}\left(x^{j}\right)$, with $x^{j} \in \overline{k(\bar{\Omega})}$. Define $\mu^{j}(x)=$ $\frac{\lambda^{i}(x)}{\sum_{j} \lambda^{j}(x)}$

Where $\quad \lambda^{i}(x)=\max \left(0, \epsilon-\left\|\mathrm{k}(\mathrm{x})-\mathrm{x}^{i}\right\|\right) \quad . \quad$ This maximum is zero whenever $\mathrm{k}(\mathrm{x}) \notin \overline{B\left(x^{l}\right)}$ and therefore $\mu^{i}(x)=0$, unless $\left\|k(x)-x^{i}\right\|<\epsilon$.

Set $\mathrm{k}^{\epsilon}(x)=\sum_{i} \mu^{i}(x) \cdot x^{i}$. Now $\mathrm{k}^{\epsilon}(\Omega) \subset \operatorname{span}\left(x^{i}\right)$. As for the approximation we obtain: $\left\|\mathrm{k}-\mathrm{k}^{\epsilon}\right\|_{C_{b}^{0}}=$ $\left\|k-\sum_{i} \mu^{i}(x) \cdot x^{i}\right\|_{C_{b}^{0}}=\left\|\sum_{i} \mu^{i}(x) \cdot\left(k-x^{i}\right)\right\|_{C_{b}^{0}}$

$$
\begin{gathered}
\left\|\mathrm{k}-\mathrm{k}^{\epsilon}\right\|_{C_{b}^{0}}=\sup _{x \in \bar{\Omega}}\left\|\sum_{i} \mu^{j}(x)\left(k(x)-x^{j}\right)\right\| \\
\leq \sup _{x \in \bar{\Omega}}\left\|\sum_{i} \mu^{j}(x)\left(k(x)-x^{j}\right)\right\|<\sum_{i} \mu^{i}(x) \epsilon=\epsilon
\end{gathered}
$$

Which complete the proof \#

\section{Preliminaries}

Definition of the Leray - Schauder degree (2-1): Let $f$ be a continuous map of the form $f \in \mathcal{C}_{I d}^{0}(\bar{\Omega})$, and let $p f \notin(\partial \Omega)$. Let $k^{\epsilon}$ be a finite rank perturbation with

$\left\|k-k^{\epsilon}\right\|_{C_{b}^{0}}<\epsilon$ and $\epsilon<\delta / 2$ ( $\delta$ as given above ) and with $k^{\epsilon}(\bar{\Omega}) \subset Y^{\epsilon} \subset X$ (subspaces). Then for any finite dimensional subspace $X^{\epsilon}$ containing both $Y^{\epsilon}$ and $p$, define the Leray Schauder degree as

$$
\operatorname{deg}(f, \Omega, p):=\operatorname{deg}\left(f^{\epsilon}, \Omega \cap X^{\epsilon}, p\right)
$$

Where $f^{\epsilon}=I d-k^{\epsilon}$. If there is no ambiguity about the context we mostly omit the subscript for the notation.

Lemma (2-2): Let $\widetilde{X^{\epsilon}} \subset X$ be any finite dimensional subspace such that $Y^{\epsilon} \subset \widetilde{X^{\epsilon}}$ and $p \in X^{\epsilon}$. Then $\operatorname{deg}\left(f^{\epsilon}, \Omega \cap\right.$ $\left.\widetilde{X^{\epsilon}}, p\right):=\operatorname{deg}\left(f^{\epsilon}, \Omega \cap X^{\epsilon}, p\right)$

Proof: Step (i): Consider mappings of the from $g=I d-$ $h: \bar{D} \subset \mathbb{R}^{n} \oplus \mathbb{R}^{m} \rightarrow \mathbb{R}^{n} \oplus \mathbb{R}^{m}$, with $h(\bar{D}) \subset \mathbb{R}^{n}$. Suppose $p \in \mathbb{R}^{n}$ and $p \notin g(\partial D)$. Then $\operatorname{deg}(g, D, P)=\operatorname{deg}\left(g_{n}, D \cap\right.$ $\left.\mathbb{R}^{n}, p\right)$, where $g_{n}=\left.g\right|_{D \cap \mathbb{R}}$

$W e$ prove the above statement in the case that $h$ is $C^{1}$, since the degree is defined via $C^{1}$ approximations and with $P=0$. Let $\omega_{1}$ and $\omega_{2}$ be top forms on $\mathbb{R}^{n}$ and $\mathbb{R}^{m}$ respectively with $\int_{\mathbb{R}^{n}} \omega_{1}=\int_{\mathbb{R}^{m}} \omega_{2}=1$ and their supports contained in a sufficiently small neighborhood of the origin. In terms of coordinates we write $x=x_{1}+x_{2}, x_{1} \in$ $\mathbb{R}^{n}$ and $x_{2} \in \mathbb{R}^{m}$. For the degree this yields

$$
\begin{gathered}
\operatorname{deg}(g, D, P)= \\
\int_{D} g^{*}\left(\omega_{1} \oplus \omega_{2}\right)=\int_{D} \omega_{1}\left(x_{1}-h\left(x_{1}+\right.\right. \\
\left.\left.x_{2}\right)\right) \omega_{2}\left(x_{2}\right) J_{g}\left(x_{1}+x_{2}\right) d x_{1} d x_{2}
\end{gathered}
$$

By the specific from of we have that $J_{g}\left(x_{1}+x_{2}\right)=$ $\operatorname{det}\left(I d-\frac{\partial h}{\partial x_{1}}(x)\right)$ since the expression for the degree is independent of $\omega_{1}$ and $\omega_{2}$ we can choose $\omega_{2}$ to approximate a density function that peaks at 0 and has integral equal to 1 approximating a delta distribution. Due to the independence on $\omega_{2}$ this give

$$
\begin{gathered}
\operatorname{deg}(g, D, P)= \\
=\int_{D \cap \mathbb{R}^{n}} \omega_{1}\left(x_{1}-h\left(x_{1}+x_{2}\right)\right) \operatorname{det}\left(i d-\frac{\partial h}{\partial x_{1}}\left(x_{1}\right)\right) d x_{1}= \\
\operatorname{deg}\left(g_{n}, D \cap \mathbb{R}^{n}, p\right)
\end{gathered}
$$

Step (ii): Since $Y^{\epsilon} \subset X^{\epsilon} \cap \tilde{X}^{\epsilon}$ and $p \in X^{\epsilon} \cap \tilde{X}^{\epsilon}$ we may assume without loss of generality that $X^{\epsilon} \cap \tilde{X}^{\epsilon}$. By construction it holds that $f^{\epsilon}: \bar{\Omega} \cap X^{\epsilon} \rightarrow X^{\epsilon}$ and

$f^{\epsilon}: \bar{\Omega} \cap \tilde{X}^{\epsilon} \rightarrow \tilde{X}^{\epsilon}$. By construction it holds that $f^{\epsilon}: \bar{\Omega} \cap$ $X^{\epsilon} \rightarrow X^{\epsilon}$ and

$f^{\epsilon}: \bar{\Omega} \cap \tilde{X}^{\epsilon} \rightarrow \tilde{X}^{\epsilon}$. Consider the linear change of variables $y=q(x)$ such that $q\left(X^{\epsilon}\right)=\mathbb{R}^{n} \oplus 0$ and $\left(\widetilde{X^{\epsilon}}\right)=\mathbb{R}^{n} \oplus \mathbb{R}^{m}$. From Step (i) it follows that ion

$\operatorname{deg}(g, D, P)=\operatorname{deg}\left(g_{n}, D \cap \mathbb{R}^{n}, p\right)$. It remains to prove that the degree is invariant under the change of coordinates. Using differential characterization of the degree we obtain:

$$
\begin{gathered}
\int_{D} g^{*} \omega=\int_{D}\left(q o f^{\epsilon} o q^{-1}\right)^{*} \omega \\
=\int_{D}\left(q^{-1}\right)^{*}\left(\left(f^{\epsilon}\right)^{*}\left(q^{*} \omega\right)\right) \\
=\operatorname{sign}\left(J_{q^{-1}}(x)\right) \int_{\bar{\Omega} \cap \widetilde{X}^{\epsilon}}\left(f^{\epsilon}\right)^{*}\left(g^{*} \omega\right) \\
=\operatorname{sign}\left(J_{q^{-1}}(x)\right) \operatorname{deg}\left(f^{\epsilon}, \Omega \cap \tilde{X}^{\epsilon}, p\right) \int_{\mathbb{R}^{n+m}} g^{*} \omega \\
=\operatorname{sign}\left(J_{q^{-1}}(x)\right)\left[\operatorname { d e g } \left(f^{\epsilon}, \Omega \cap\right.\right. \\
\left.\left.\tilde{X}^{\epsilon}, p\right)\right] \operatorname{sign}\left(J_{q}(y)\right) \int_{\mathbb{R}^{n+m}} \omega \\
=\operatorname{deg}\left(f^{\epsilon}, \Omega \cap \tilde{X}^{\epsilon}, p\right) \int_{\mathbb{R}^{n+m}} \omega
\end{gathered}
$$

Since $\int_{D} g^{*} \omega=\operatorname{deg}\left(g, D, q(p) \int_{\mathbb{R}^{n+m}} \omega\right.$ it follows that:

$$
\operatorname{deg}\left(g, D, q(p)=\operatorname{deg}\left(f^{\epsilon}, \Omega \cap \tilde{X}^{\epsilon}, p\right)\right.
$$

Which proves that the degree is invariant under coordinate changes.

By restricting to the subspace $\epsilon$ we obtain $\operatorname{deg}\left(g_{n}, D \cap\right.$ $\left.\mathbb{R}^{n}, q(p)\right)=\operatorname{deg}\left(f^{\epsilon}, \Omega \cap \tilde{X}^{\epsilon}, p\right)$. The proof follows now from Step (i).

Lemma (2-3): Let $k^{\epsilon}$ and $\tilde{k}^{\epsilon}$ both be finite rank approximations for with $\left\|k-k^{\epsilon}\right\|_{C_{b}^{0}}<\epsilon\left\|k-k^{\epsilon}\right\|_{C_{b}^{0}}<\epsilon$ 
and $\epsilon<\delta / 2$. Then

$$
\operatorname{deg}\left(I d-k^{\epsilon}, \Omega \cap X^{\epsilon}, p\right)=\operatorname{deg}\left(I d-\tilde{k}^{\epsilon}, \Omega \cap \tilde{X}^{\epsilon}, p\right)
$$

For any subspace $X^{\epsilon}$ and $\tilde{X}^{\epsilon}$ containing both $p$ and the ranges of $k^{\epsilon}$ and $\tilde{k}^{\epsilon}$

Respectively.

Proof:

Let $Z^{\epsilon} \subset X$ be a finite dimensional linear subspace containing both $X^{\epsilon}$ and $\tilde{X}^{\epsilon}$

From Lemma (2-3) it follows that

$$
\begin{aligned}
& \operatorname{deg}\left(I d-k^{\epsilon}, \Omega \cap X^{\epsilon}, p\right)=\operatorname{deg}\left(I d-k^{\epsilon}, \Omega \cap Z^{\epsilon}, p\right) \\
& \operatorname{deg}\left(I d-\tilde{k}^{\epsilon}, \Omega \cap \hat{X}^{\epsilon}, p\right)=\operatorname{deg}\left(I d-\tilde{k}^{\epsilon}, \Omega \cap Z^{\epsilon}, p\right)
\end{aligned}
$$

Consider the compact homotopy $k_{t}^{\epsilon}=(1-t) k^{\epsilon}+t \tilde{k}^{\epsilon}$. which yields a homotopy $f_{t}^{\epsilon}=I d-k_{t}^{\epsilon}$ and is a proper homotopy.

Then $\operatorname{deg}\left(I d-k^{\epsilon}, \Omega \cap Z^{\epsilon}, p\right)=\operatorname{deg}\left(I d-\tilde{k}^{\epsilon}, \Omega \cap Z^{\epsilon}, p\right)$ which then prove that $\operatorname{deg}\left(I d-k^{\epsilon}, \Omega \cap X^{\epsilon}, p\right)=\operatorname{deg}(I d-$ $\left.\tilde{k}^{\epsilon}, \Omega \cap \tilde{X}^{\epsilon}, p\right)$

The Leray -Schauder degree is well defined.

Theorem (2-4): Let $\mathrm{F}$ be $C^{\infty}-$ function $\bar{\Omega} \times R^{m}$ which defined a strongly elliptic nonlinear partial differential operator $F(u)$ of order $2 m$ on $\Omega$. Let $0<\lambda<1$ and for $0 \leq t \leq 1$, let $F(x, p, t)=t F(x, p)+(1-t) \sum_{|\alpha|}\left|p_{\alpha}\right|^{2}$, $F_{t}(u)$ the corresponding

The corresponding partial differential operator of order. Suppose that all of the following conditions are satisfied:

For each $>0$, there exists constant $\mu$ with $0<\mu<\lambda<1$ and a differential operator $H$ of order $\leq 2 m-1$ "possibly nonlinear" on $\Omega$ such that for each $\mathrm{u} \in \mathrm{C}_{0}^{2 m-1, \lambda}(\Omega), \mathrm{v} \in$ $\mathrm{C}_{0}^{2 m, \mu}(\Omega)$ with $\|\mathrm{u}\|_{o^{2 m-1, \lambda}} \leq R$ and

$$
\begin{gathered}
\left\{p_{x}(u, v)\right\}_{\alpha}=\left\{\begin{array}{l}
D^{\alpha} u(x),|\alpha|<m \\
D^{\alpha} v(x),|\alpha|=m
\end{array}\right. \text { the linear equation } \\
\left.\left.\sum_{|\alpha|=2 m} F_{t \alpha}\left(x, p_{x}(u, v)\right)\right) D^{\alpha} \eta+\sum_{|\alpha|=2 m-1} t H_{\alpha}\left(x, p_{x}(u)\right)\right) D^{\alpha} \eta=0
\end{gathered}
$$

Has only $\eta=0$ as a solution in $\mathrm{C}_{0}^{2 m, \mu}(\Omega), 0 \leq t \leq 1$.

For given $R>0$ and the corresponding function $H$ of condition (1), there exists a function $R_{1}(s)$ such that for $u$ in $\mathrm{C}_{0}^{2 m-1, \lambda}(\Omega)$ with $\|\mathrm{u}\|_{o^{2 m-1, \lambda}(\Omega)} \leq R$ and any

$v$ in $\mathrm{C}_{0}^{2 m, \lambda}(\Omega)$ such that $p_{x}(u, v)+t H_{\alpha}\left(p_{x}(u)\right)=f$, for some $t$ in $[0,1]$ and $f \in \mathrm{C}^{0, \lambda}(\Omega)$ with $\|f\|_{0^{0}, \lambda(\Omega)} \leq s$, we have $\|v\|_{{ }^{0}, \lambda(\Omega)} \leq R_{1}(s)$

There exists a constant $R_{0}>0$ such that for any $t$ in $[0,1]$

$$
\begin{gathered}
\left.\left.F_{t}(p(u, v))+t H_{t} p(v)\right)-t H_{t} p(u)\right) \\
\left.=F_{t_{0}}\left(p\left(u_{0}, v\right)\right)-t_{0} H_{t_{0}} p(v)\right)+\left\{F_{t_{0}}(p(u, v))-F_{t_{0}}\left(p\left(u_{0}, v\right)\right)\right\} \\
\left.\left.\left.\left.+\left\{t H_{t} p(v)\right)-t_{0} H_{t_{0}} p(v)\right)\right\}-t_{0} H_{t_{0}}\left(p\left(u_{0}\right)\right)+\left\{t H_{t} p(v)\right)-t_{0} H_{t_{0}} p(u)\right)\right\}
\end{gathered}
$$

Furthermore

$$
F_{t_{0}}\left(p\left(u_{0}, v\right)\right)=F_{t_{0}}\left(p\left(u_{0}, v\right)\right)+\grave{F}_{t_{0}}\left(p\left(u_{0}, v\right)\right)\left(u-v_{0}\right)+R\left(u_{0}, t_{0}, v\right)
$$
equation $(i)$ is

$F_{t_{0}}\left(p\left(u_{0}, v\right)\right)=F_{t_{0}}\left(p\left(u_{0}, v\right)\right)+\grave{F}_{t_{0}}\left(p\left(u_{0}, v\right)\right)\left(u-v_{0}\right)+R\left(u_{0}, t_{0}, v\right)$ and in $v \in C_{0}^{2 m, \mu}(\Omega)$, if

Then the equation $F(u)=0$ has a solution $u \in C_{0}^{2 m, \mu}(\Omega)$.

Proof of Theorem: Let $R=R_{0} . B=\left\{u \mid u \in C_{0}^{2 m, \mu}(\Omega),\|u\| \leq R\right\}$. Let $H$ be the function corresponding to $R$ by condition (1) of the hypothesis of Theorem (2-1).

For each $u$ in $R_{R}$, we consider the equation:

$$
\text { (i) } \left.\left.F_{t}(p(u, v))+t H p(v)\right)=t H p(u)\right)
$$

For $v$ in $\in C_{0}^{2 m, \mu}(\Omega)$. The linearized form of

$$
\text { (ii) } \left.\left.\sum_{|\alpha|=2 m} F_{t, \alpha}(u, v)\right) D^{\alpha} \eta+\sum_{|\alpha|=2 m-1} t H_{\alpha}(p, v)\right) D^{\alpha} \eta
$$

Which by condition (1) has only $\eta=0$ as a solution in $\in C_{0}^{2 m, \mu}(\Omega)$ for a fixed $\mu$ with $0<\mu<\lambda<1$. Moreover by condition (ii) of the hypothesis, the solution $v$ of the equation

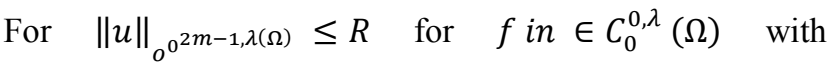
$\|f\|_{o^{o, \lambda}} \leq s$, where $v$ lies in $\mathrm{C}_{0}^{2 m, \lambda}(\Omega)$, must satisfy the inequality $\|v\|_{o}{ }_{2 m-1, \lambda(\Omega)} \leq R_{1}(s)$. Hence the hypotheses of Theorem (2-1) are satisfied for the family of equations (iii) and in particular, equation $(i)$ has one and only one solution $v_{t}$ for each $t$ in $[0,1]$.

set $C_{t}(u)=v_{t}$. Then $C_{t}$ is a well-defined mapping on whose range we consider as a subset of $C_{0}^{2 m-1, \lambda}(\Omega)$. Since the map $u \rightarrow H(p(u))$ carries bounded sets of $\mathrm{C}_{0}^{2 m-1, \lambda}(\Omega)$ into bounded sets of $\mathrm{C}_{0}^{0, \lambda}(\Omega)$ it follows from the argument of the preceding paragraph that $\left\|\mathrm{C}_{t} \mathrm{u}\right\|_{o^{2 m-1, \lambda}(\Omega)} \leq$ $R_{2}$ for all $u$ in $B_{R}$ and all $t$ in $[0,1]$ with a fixed constant $R_{2}>0$. Since $\mathrm{C}_{0}^{2 m, \lambda}(\Omega)$ has a compact injection into $\mathrm{C}_{0}^{2 m-1, \lambda}(\Omega)$, it follows that $\mathrm{U}_{0 \leq t \leq 1} C_{t}\left(B_{R}\right)$ is precompact in $\mathrm{C}_{0}^{2 m-1, \lambda}(\Omega)$

We wish now to verify that mapping $[t, u] \rightarrow C_{t}(u)$ is a continuous mapping of

$[0,1] \times B_{R}$ into $\mathrm{C}_{0}^{2 m-1, \lambda}(\Omega)$. Let $t_{0}$ be a fixed number in $[0,1], u_{0}$, we have 
Where

$$
v_{0}=C_{t_{o}}\left(v_{0}\right) \text { and }\left\|R_{1}\left(u_{0}, v_{0}, t_{0}, v\right)\right\|_{o^{0, \lambda}}=0\left(\left\|u-v_{0}\right\|\right) \text { as }\left\|u-v_{0}\right\|_{o^{2 m, \lambda}} \rightarrow 0
$$

The norm of $u-v_{0}$ will be taken in $\mathrm{C}_{0}^{2 m, \lambda}(\Omega)$ throughout this argument.

Similarly

$$
t_{0} H_{t_{0}}(p(v))=t_{0} H_{t_{0}}\left(p\left(v_{0}\right)\right)+t_{0} \grave{H}_{t_{0}}\left(p\left(v_{0}\right)\right)\left(u-v_{0}\right)+R_{1}\left(u_{0}, v_{0}, t_{0}, v\right)
$$

Where $R_{1}\left(u_{0}, v_{0}, t_{0}, v\right)=0\left(\left\|u-v_{0}\right\|\right)$ as $\left\|u-v_{0}\right\|_{o^{2 m, \lambda}} \rightarrow 0$

It follows that for $v$ near $v_{0}$ in $\mathrm{C}_{0}^{2 m, \lambda}(\Omega)$ we have

$$
\begin{gathered}
\left.\left.F_{t}(p(u, v))+t H_{t} p(v)\right)-t H_{t} p(u)\right)=\left\{F_{t_{0}}\left(p\left(u_{0}, v_{0}\right)\right)+t_{0} H_{t_{0}}\left(p\left(u_{0}\right)\right)-t_{0} H_{t_{0}}\left(p\left(v_{0}\right)\right)\left[\grave{F}_{t_{0}}\left(p\left(u_{0}, v_{0}\right)\right)\right.\right. \\
\left.+t_{0} \grave{H}_{t_{0}}\left(p\left(u_{0}\right)\right)\right]\left(u-v_{0}\right)+R_{2}\left(u_{0}, v_{0}, t_{0}, t, u, v\right)
\end{gathered}
$$

Where $\left\|R_{2}\left(u_{0}, v_{0}, t_{0}, t, u, v\right)\right\|_{o^{2 m-1, \lambda}(\Omega)}$

$$
\leq \sigma\left(u-v_{0}\right)\left\|u-v_{0}\right\|_{o^{2 m, \lambda}}+\sigma_{3}\left(u_{0}, v_{0}, t_{0}, t, u, v\right)
$$

And $\sigma_{3}\left(u_{0}, v_{0}, t_{0}, t, u, v\right) \rightarrow 0$ as $\left\|u-v_{0}\right\|_{o^{2 m, \lambda}}+\left|t-t_{0}\right| \rightarrow 0$

The condition that $\left.(i) p(u, v))+t H_{t} p(v)\right)=$ $\left.t H_{t} p(u)\right)=0$

Can therefore be satisfied if

$$
\left.\left[\grave{F}\left(p\left(u_{0}, v_{0}\right)\right)+t_{0} \grave{H}_{t_{0}} p\left(v_{0}\right)\right)\right]=-R_{2}\left(u_{0}, v_{0}, t_{0}, t, u, v\right)
$$

The operator in square brackets is an isomorphism of $\mathrm{C}_{0}^{2 m, \lambda}(\Omega)$ with $\mathrm{C}^{0, \lambda}(\Omega)$ by

Condition (1) of the hypothesis. Hence for $\left\|u-v_{0}\right\|+$ $\left|t-t_{0}\right|$ sufficiently small, we may find a solution $v$ of equation $(i)$ in a prescribed neighborhood of $v_{0}$ in $\mathrm{C}_{0}^{2 m, \lambda}(\Omega)$ with $\left\|u-v_{0}\right\|_{o^{2 m, \lambda}} \leq \rho\left(\left\|u-v_{0}\right\|+\left|t-t_{0}\right|\right)$

Where $(s) \rightarrow 0$ as $s \rightarrow 0$. Since the solution of $(i)$ is unique, $v=C_{t}(u)$. Hence $C_{t}$ maps $[0,1] \times B_{R}$ continuously into $C_{0}^{2 m, \lambda}(\Omega)$ and a fortiori into $C_{0}^{2 m-1, \lambda}(\Omega)$.

We now apply the theory of the Leray - Schauder degree to the family of mappings

$I-C_{t}, 0 \leq t \leq 1$. For $t=1, C_{t}=0$ since then $v$ is a solution of

$$
\sum_{|\alpha|=m} D^{\alpha} D^{\alpha} v=0
$$

Hence the degree of $T_{0}$ over $B_{R}$ with respect to 0 is equal to +1 . For each $t$ in $[0,1], C_{t}$

Is a compact map and the degree of $T_{t}$ over $B_{R}$ with respect to 0 is well - defined since

For $u$ in $B_{R}$ with $\|u\|_{o} 2 m, \lambda(\Omega)=R, T_{t} u=0$ implies that

$$
\left.\left.F_{t}(p(u))+t H p(v)\right)=t H p(u)\right) \text { i.e } F_{t}(p(u))=0
$$

And for solutions of the latter equation. condition (3) of the hypothesis assures that

$\|u\|_{o^{2 m, \lambda(\Omega)}} \leq R_{0}=R$. The degree of $T_{t}$ over $B_{R}$ with respect to 0 is constant in $t$ by the continuity and compactness of $C_{t}$ in the pair $[t, u]$. Hence the degree of $T_{1}$ over $B_{r}$ with respect t 0 ois equal to +1 and there exists a solution $u$ in $B_{R}$ of $T_{1} u=0$.

This is equivalent, however to $F(p(u))=0$ \#
The problem with degree theory in infinite dimensional spaces is that homotopy

Invariance, a basic property of the degree, prevents the existence of a nontrivial

Degree theory. We can alter the notion of homotopy invariance in order to a degree theory, or limit the types of maps for which a degree is well defined the Leray Schauder degree does both by considering specific types of mappings. Namely mappings of the form $f=I d-k$, where $I d$ is the identity map on $X$ and

$\mathrm{k} \in \mathrm{K}(\bar{\Omega})$. Homotopies are considered in the same class, denote the function class by $\mathcal{C}_{I d}^{0}(\bar{\Omega})=\{f=I d-$ $k \mid k \in K\}$ and by $\mathcal{C}_{I d}^{0}(\bar{\Omega})$ for mapping defined on $X$

Properties of the Leray - Schauder degree:

Theorem (2-5): For Leray -Schauder degree we have the following properties:

(A1) if $p \in \Omega$, then $\operatorname{deg}_{L S}(I d, \Omega, p)=1$

(A2) for $\Omega^{1}, \Omega^{2} \subset \Omega$, disjoint open subsets of $\Omega$, and then $\mathrm{p} \notin \Omega^{1} \cup \Omega^{2}$, it holds that

$$
\operatorname{deg}_{L S}(f, \Omega, p)=\operatorname{deg}_{L S}\left(f, \Omega^{1}, p\right)+\operatorname{deg}_{L S}\left(f, \Omega^{2}, p\right) ;
$$

(A3) for any continuous path $t \rightarrow f_{t}=I d-k_{t}, k_{t} \in K(\bar{\Omega})$ and $t \rightarrow p_{t}$, with $p_{t} \notin f_{t}(\partial \Omega)$ it holds that $\operatorname{deg}_{L S}\left(f_{t}, \Omega, p_{t}\right)$ is independent of $\in[0,1]$; $\operatorname{deg}_{L S}$ and is called a degree theory.

As in the case of the Brouwer degree the essential properties of the Leray -Schauder degree follow from (A1) (A2).

Property (Validity of the degree) (2-6): If $p \notin \Omega$, the $\operatorname{deg}_{L S}(f, \Omega, p)=0$. Conversely, if then $\operatorname{deg}_{L S}(f, \Omega, p) \neq 0$, then there exists a $x \in \Omega$ such that $f(x)=p$.

Property (Continuity of the degree) (2-7): The degree $\operatorname{deg}_{L S}(f, \Omega, p)$ is continuous in $f=I d-k$, i.e there exists a $\delta=\delta(p, f)>0$, such that for all $g=I d-\tilde{k}$ satisfying $\|k-\tilde{k}\|_{C_{b}^{0}}<\delta \quad, \quad$ it holds that $p \notin g(\partial \Omega)$ and $\operatorname{deg}_{L S}(g, \Omega, p)=\operatorname{deg}_{L S}(f, \Omega, p)$.

Property (Dependence on path components) (2-8): The degree only depends on the path components $D \subset X \backslash f(\partial \Omega)$ i.e for any two points $p, q \in D \subset X \backslash f(\partial \Omega)$ it holds that $\operatorname{deg}_{L S}(f, \Omega, p)=\operatorname{deg}_{L S}(f, \Omega, D)$ this justifies the notation $\operatorname{deg}_{L S}(f, \Omega, D)$.

Property (Translation invariance) (2-9): The degree is 
invariant under translation, i.e. for any $q \in X$ it holds that $\operatorname{deg}_{L S}(f-q, \Omega, p-q)=\operatorname{deg}_{L S}(f, \Omega, p)$

Property (Excision) (2-10): Let $\Lambda \subset \Omega$ be a closed subset in $\Omega$ and $p \notin f(\Lambda)$. Then $\operatorname{deg}_{L S}(f, \Omega, p)=\operatorname{deg}_{L S}(f, \Omega, \backslash \wedge, p)$

Property (Additivity) (2-11): Suppose tha $\Omega^{i} \subset \Omega, i=$ $1, \ldots, k$ are disjoint open subsets of $\Omega$, and $p \notin \mathrm{f}(\bar{\Omega} \backslash$ $\left.\left(\cup_{i} \Omega_{i}\right)\right)$, then $\operatorname{deg}_{L S}(f, \Omega, p)=\sum_{i} \operatorname{deg}_{L S}\left(f, \Omega^{i}, p\right)$

Definition of the Compact homotopies (2-12): Two mappings

$f, g: \bar{\Omega} \subset X \rightarrow Y$ are said to be compactly homotopic relative of $F$ is there exists a family of compact mappings: $k(t,):. \bar{\Omega} \subset X \rightarrow Y, t \in[0,1]$, such that

$$
\begin{aligned}
& h(t, x)=F(x)+k(t, x) \\
& h(0, x)=F(x)+k(0, x)=f(x) \\
& h(1, x)=F(x)+k(1, x)=g(x)
\end{aligned}
$$

The associated compact homotopy classes are denoted by $[f]_{c}$.

\section{Main Result: Semi Linear Elliptic Equations and a Priori Estimates}

In this section we will give Application of the Leray Schauder degree in the context of nonlinear elliptic equations. We follow the notes by L. Nirenberg.

The methods that we discuss apply in general for elliptic differential operator of any order. Let $D \subset \mathbb{R}^{n}$ be a bounded domain with smooth boundary $\partial D$.

Consider the problem $-\Delta u=g(x, u, \nabla u), u=0, x \in \partial D$

For the nonlinearity $g$ we assume that $C^{\infty}-$ function of arguments, i.e $g \in C^{\infty}\left(\bar{D} \times \mathbb{R} \times \mathbb{R}^{n}\right)$, and $|g(x, u, \nabla u)| \leq$ $C+C|\nabla u|^{\gamma}, \gamma<1$

Uniformly in $x \in \bar{D}$ and $u \in \mathbb{R}$. Under these conditions we can prove the following result.

Theorem (3-1): Under the assumptions on $g$ the above elliptic equations has a solution $u \in C^{\infty}(\bar{D})$. Moreover, if $g(x, 0,0) \not \equiv 0$, then the solution $u$ is not identically zero.

Proof: The idea behind the proof is the formulate the above elliptic equation as a problem of finding zeroes of an appropriate function $f$ on (infinite dimensional ) Banach space.

Let start with choosing an appropriate space in which to work. Define $X=H^{2} \cap H_{0}^{1}(D)$ to be the intersection of two Soblev space.

The space $H^{2} \cap H_{0}^{1}(D)$ is a Hilbert space with norm $\|u\|_{x}=\int_{D}|\Delta u| d x$

Due to the Dirichlet boundary conditions the Laplace operator

$-\Delta: H^{2} \cap H_{0}^{1}(D) \subset L^{2}(D) \rightarrow L^{2}(D)$ has a compact inverse $(-\Delta)^{-1}: L^{2}(D) \rightarrow L^{2}(D)$

We rewrite the elliptic equation as:

$$
u-(-\Delta)^{-1} g(x, u, \nabla u)=0
$$

The above equation can be regarded as a seeking zeroes of the (Nemytskii)
Mapping $f(u)=u-(-\Delta)^{-1} g(x, u, \nabla u)$ on $H^{2} \cap$ $H_{0}^{1}(D)$. By the estimate on $g$ we have that

$$
\begin{gathered}
\int_{D}|g(x, u(x), \nabla u(x))|^{2} d x \leq C \int_{D}\left[1+|\nabla u(x)|^{2 \gamma}\right] d x \\
\leq \grave{C}\left(\int_{D}\left[1+|\nabla u(x)|^{2 \gamma}\right] d x\right)^{\gamma} \\
\leq C\left(1+\|u\|_{H_{0}^{1}}^{2}\right)^{\gamma}
\end{gathered}
$$

Which prove that for $u \in X, g(x, u, \nabla u)(x)$. Is an $L^{2}-$ function. Consequently, t he

Composition $(-\Delta)^{-1}[g(x, u, \nabla u)] \in X$, proving that $f: X \rightarrow X$ is well defined. The latter follows from the fact that $R\left((-\Delta) i^{-1}=H^{2} \cap H_{0}^{1}(D)\right.$. As a map from $L^{2}$ to $H^{2} \cap$ $H_{0}^{1}(D)$, the inverse Laplacian is an isometry. Concerning the continuity of this substitution map. If we define $Y=H_{0}^{1}(\Omega)$ then $f$ is a map from $Y$ to $Y$, and $f=i d-k$, where $k: Y \rightarrow$ $Y$ is a compact map. Indeed, $k$ is a composition of the Nemytskii map $u \mapsto g(x, u, \nabla u)$ (from $Y$ to $L^{2}$ ), the inverse Laplacian $(-\Delta)^{-1}$ (from $L^{2}$ to $X$ ), and the compact embedding $X \hookrightarrow Y$, which proves the compactness of $k$. This brings us into the realm of the Leray - Schauder degree.

Suppose $u \in X$ is a solution of the equation (3-1-1), then the estimate on $g(x, u, \nabla u)$ can be used now to obtain an a priori estimate on the solutions.

$$
\begin{gathered}
\|u\|_{Y}^{2} \leq C \\
\leq\left(1+\|u\|_{X}^{2}=C\|g(x, u, \nabla u)\|_{L^{2}}^{2}\right)^{\gamma} \\
\leq(1+
\end{gathered}
$$

Which, since $\gamma<1$, implies that $\|x\|_{Y} \leq R$

Define the domain $\Omega=B_{2 R}(0) \subset X$. Clearly, $f$ is a continuous map from $\bar{\Omega}$ into $X$, which is of the from identity minus compact. Due to the above a priori estimate $f^{-1}(0) \subset$ $B_{R}(0)$ and $0 \notin f_{t}(\partial \Omega)$ and therefore the Leary -Schauder degree $\mathrm{d}_{L S}(\mathrm{f}, \Omega, 0)$ is well defined.

In order to compute this degree we consider the following homotopy:

$$
f_{t}(u)=u-t(-\Delta)^{-1}[g(x, u, \nabla u)], t \in[0,1]
$$

Notice, for $t \in[0,1]$ we have via the same a priori estimates, that $f^{-1}(0) \subset B_{R}(0)$, and therefore $0 \notin$ $f_{t}(\partial \Omega)$ for all $t \in[0,1]$. Homotopy invariance of the Leary Schauder degree then yields

$$
d(f, \Omega, 0)=d(i d, \Omega, 0)=1
$$

Which implies, by validity property of the Leary Schauder degree, that $f^{-1}(0) \neq 0$. Equation (3-1-1) thus has a solution $u \in Y$. The equation yields $u=$ $(-\Delta)^{-1}[g(x, u, \nabla u)] \in X$, which that the solution also lies in $X$. To prove regularity we use a bootsrappind argument. The integral estimates on $g$ can be adjusted to $L^{P}$ estimates. This gives, by the Sobolev embeddings that:

$$
u \in H^{1, p} \Rightarrow g(x, u, \nabla u) \in L^{P} \Rightarrow u \in H^{2, p} \Rightarrow u \in H^{1, \grave{p}}
$$


Where $\frac{1}{\grave{p}}=\frac{1}{p}-\frac{1}{n}$, provide $n>p$. This yields the recurrence relation

$$
\frac{1}{p^{k+1}}=\frac{1}{p^{k}}-\frac{1}{n}
$$

We can repeat these recurrent steps until $k$ time until $2(k+1)>n>2 k$

And then $\in H^{2, p k}$, where $p_{k}=\frac{2 n}{n-2 k}$. A gain by the Sobolev embeddings, we have that $H^{2, p k}(D) \hookrightarrow C^{1, \alpha}(\bar{D})$.

Where $\alpha=1-\frac{n}{p^{k}}$, since $\frac{n}{p^{k}}=\frac{n}{2}$, and $k+1>\frac{n}{2}>k$, it holds $0<\alpha<1$ We now repeat the bootsrappind in the Holder space:

$$
u \in C^{1, \alpha} \Rightarrow g(x, u, \nabla u) u \in C^{0, \gamma \alpha} \Rightarrow u \in C^{2, \grave{\alpha}}
$$

Where $\grave{\alpha}=\gamma \alpha$. The idea now is the use the elliptic regularity theory for the Laplacian be differentiation the equation. Let $v_{i}=\frac{\partial u}{\partial x_{i}}$ then

$$
-\Delta v_{i}=\partial_{x_{i}} g+\left(\partial_{u} g\right) v_{i}+\sum_{j} \partial_{v_{j}} g \frac{\partial v_{j}}{\partial x_{i}}
$$

Singe $g$ is $C^{\infty}$ function of its arguments, and $u \in C^{2, \grave{\alpha}}$, the right hand side is in $C^{0, \grave{\alpha}}$, implying that $v_{i} \in C^{2, \grave{\alpha}}$, and thus $u \in C^{3, \grave{\alpha}}$. We can repeat this process indefinitely, which proves that $u \in C^{\infty}(\bar{D})$

If $g(x, 0,0) \not \equiv 0$, then $u=0$ cannot be a solution, and thus $u \neq \equiv 0$ \#

\section{Acknowledgements}

I would like to express my thanks to an anonymous referee for useful comments.

\section{References}

[1] R. C. A. M. Vandervorst, Topological Methods for Nonlinear Differential Equations, April 25, 2008.

[2] R. A. Adams and J. J. Fournier, Sobolev space, $2^{\text {nd }}$. pure and Applied Mathematics, Vol 140, Elsever/Academic Press, Amsterdam, 2003.

[3] Y. Andre, Series Gevery de type arithnetique I. Theoremes de purete et de dualite, Annals of Mathematics 151, (2000).

[4] L. C. Evans, Partial differential equations, Graduate Studies in Mathematics, Vol 19, American Mathematical Society, Providence, RI, 1998.

[5] Felix E. Browder, Topological Methods for nonlinear Elliptic Equations of Aritary order. Pacific Journal of Mathematics, Vol. 17, n 01. 1966.

[6] J. Cronin, Fixed points and topological degree in nonlinear analysis, Providence, 1964.

[7] F. E. Browder, On the spectral theory of elliptic differential operators, J, Math. Annalen, 142 (1961), 22-130

[8] M. A. Krasnoselski, Topological methods in the theory of nonlinear integral equations, Moscow, 1956 "Trans onto English, Pergamon Press, 1964".

[9] C. Miranda, Equazioni alle Derivate Parziali di Tipo Ellitic, Springer, Berlin, 1955.

[10] L. Nirenberg, Nonlinear elliptic partial differential equations and Holder continuity, Comm, Pure App. Math 6 (1953) 103157.

[11] J. Schauder, Der Fixpunktsatz in Funktionalraumen, Studia Math. 2 (1930), 171-180. 